\title{
Primer on Coupling Collective Electronic Oscillations to Nuclei
}

\author{
J. C. Solem
}

L. C. Biedenharn, Jr."

\section{DISCLAIMER}

\begin{abstract}
This report was prepared as an account of wark sponsared by an agency of the United States Government. Neither the United States Government nor any agency thereo!, nor any of their employecs, makes any warranty. express or implied, or assumes any legal liability ur responsibility for the accuracy, completeness, or usefulness of any aformatioil, apparatus, product, or process disclosed, or represents that its use would not infringe privately owned rights. Reference herein to any specific commercial product, process, or service by trade name, trademark. manufacturer, or otherwise does not necessarily constitute or imply its endorsement. recummendation, or favoring by the United States Government or any agency thereci. The views and opinions of authors expressed herein do not necessarily state or reflect those of the United States Goverriricnt or any agincy thereof
\end{abstract}




\title{
PRIMER ON COUPLING COLLECTIVE ELECTRONIC OSCILLATIONS TO NUCLEI
}

\author{
by
}

J. C. Solem and L. C. Biedenharn, Jr.

\begin{abstract}
On the basis of simple heuristic models, we show that atomic electrons can (1) amplify fields observed at the nucleus, (2) generate harmonics, and (3) drive higher multipolarities. We consider a model with the aucleus at the focus of a uniformly charged ellipsoid. It amplifies an oscillating external electric field and produces an oscillating electricfield gradient but no higher derivatives. The electric field has only odd harmonics and the electric-field gradient has only even harmonics. There is an optimum intensity for driving each harmonic.

We comment on the relevance of these results to the $U^{235}$ experiment and to the gamma-ray laser.
\end{abstract}

\section{INTRODUCTION}

Much is said about using electronic shell collective oscillation to drive a nuclear transition, but we still find misunderstanding of that process" underlying principles. In this primer we attempt to justify qualitatively and semiquantitatively three major assertions ("articles of "aith"):

1. Atomic electrons can amplify an external electromagnetic field as observed at the nucleus.

2. The electrons can produce harmonics of the external field. inducing nuclear transitions of energy greater than the quantum energy of the radiation.

3. The electrons can cause an oscillating electric field to produce nuclear transitions of multipolarity higher than that of the (dipole) source.

The first article of faith results from the mechanics of the electron in tion. The others require nonlinearity: the second requires temporal nonlinearity and the third. spatial nonlinearity. 


\section{A SIMPLE LINEAR MODEL}

To begin, consider an extremely simple model of the atom as shown in Fig. 1. The atom consists of a spherical cloud of charge with a fixed nucleus. Every electron in the cloud sees the same electric field:

$$
E_{l c s e r}=E_{n} \sin \omega t \text {, }
$$

where $\omega$ is the laser frequency. The spherical cloud is displaced from the fixed nucleus by a distance

$$
x=\iint \frac{e E_{0} \sin \omega t}{m} d^{2} t=-\frac{e E_{0} \sin \omega t}{m \omega^{2}},
$$

where e and $\mathrm{m}$ are the electron's charge and mass. Every electron has the "quiver energy" and undergoes the "quiver displacement." Now the electric field from a uniformly charged sphere acts as though all its charge were at the center. Furthermore. no field exists inside a uniformly eharged spherical shell. Therefore, the field at the nucleus owing to the electron displacement is

$$
F_{\text {ed }}=\frac{\frac{4}{3} \pi x^{3} \rho}{x^{2}}=\frac{Z e x}{r_{0}^{3}} .
$$

where $\rho$ is the cherge density (i.e., $\rho=Z e / V=3 Z e / 4 \pi r_{0}^{3}$ ). The total field at the nucleus is $E_{n u c}=E_{e d}-E_{l a s e r}$; therefore,

$$
E_{n u c}=\frac{Z e^{2} E_{0} \sin \omega t}{m \omega^{2} r_{0}^{3}}-E_{0} \sin \omega t
$$

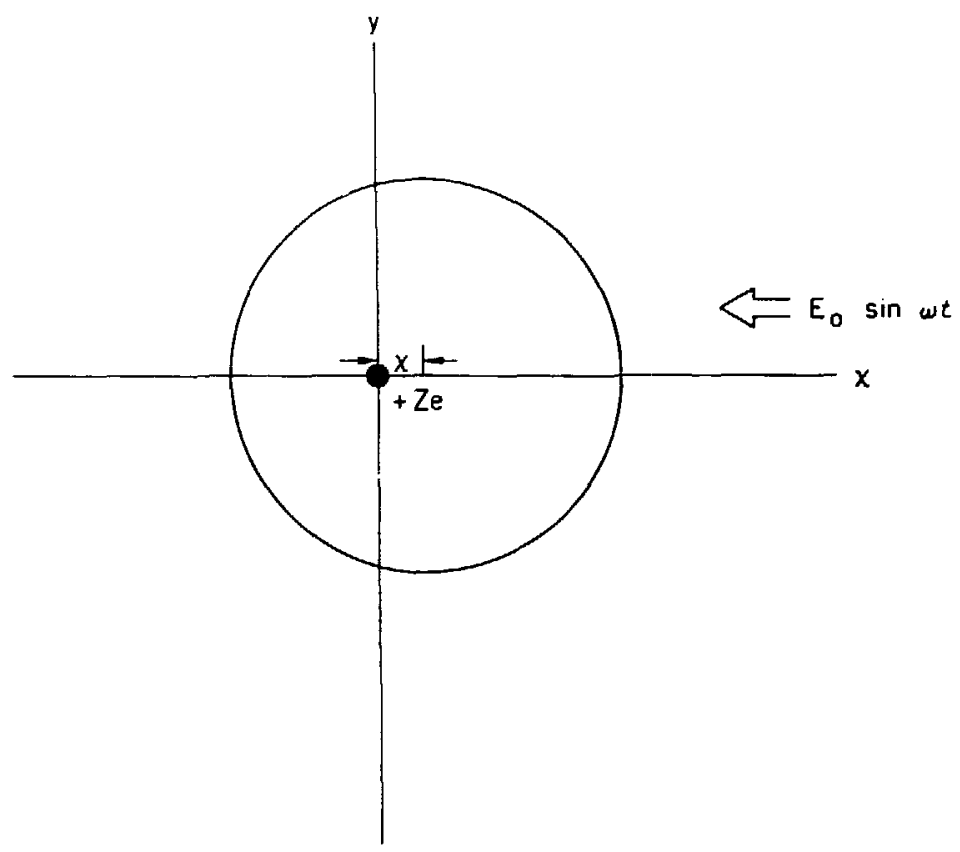

Flg. 1. Atomic electrons represented by a uniformly charged sphere. The electric field displaces the sphere from the nucleus. 
The external field is amplified by a factor

$$
A=\frac{Z e^{2}}{m \omega^{2} r_{0}^{3}}-1=\frac{Z r_{e} \star^{2}-r_{0}^{3}}{r_{0}^{3}} .
$$

where $\lambda=\lambda / 2 \pi=\mathrm{c} / \omega$ and $r_{e}$ is the classical electron radius. Taking $\lambda=193 \mathrm{~nm}$ for the experimental setup by Rhodes ${ }^{1}$ et al. and $r_{0}=1 \AA$, we get

$$
A \equiv 2.7 Z-1
$$

For uranium, the electric field at the nucleus is about 250 times the laser electric field. Therefore, we have verified, in a simple model. the first article of faith!

The manifestly linear response, as illustrated by Eq. (4), produces no harmonics, and the model does not support Article 2.

Equation (3) shows that the derivative of the field, $\frac{\partial E_{e d}}{\partial x}$, does not vanish at the nucleus, but this does not inean that a quadrupole field exists as at the nucleus. Relative to the center of the electron cloud, the potential is clearly a monopolar field:

$$
V\left(\mathbf{r}_{1}\right)=\left(\frac{Z e}{2 \mathbf{r}_{0}^{3}}\right)\left(3 r_{0}^{2}-\mathbf{r}_{1}^{2}\right),\left|\mathbf{r}_{1}\right| \leqq r_{0} .
$$

Relative to a displaced center (the position of the nucleus),

$$
V(\rho, \theta)=\left(\frac{Z_{e}}{2 \tau_{0}^{3}}\right)\left[\left(3 r_{0}^{2}-x^{2}-\rho^{2}\right)-2 x \rho \cos \theta\right] .
$$

We see from Eq. (7b) that the potential at the nucleus is a combination of monopolar and dipolar terms. In particular, all quadrupole and higher multipole terms vanish at the nucleus; therefore, the model does not support Article 3.

As a point of digression, it is interesting to note that although we have neglected the restoring forces on the electron cloud in Eq. (2) for simplicity, Eq. (3) provides a linear restoring force binding the electron cloud to the nucleus. The natural frequency is given by

$$
\omega_{0}=\sqrt{\frac{k}{m}}=\sqrt{\frac{Z^{2} e^{2}}{Z m r_{0}^{3}}}=\sqrt{\frac{Z r_{c} c^{2}}{r_{0}^{3}}} .
$$

For $Z=38$ (Krypton), this is very nearly the frequency of the laser in the experiment of Rhodes ${ }^{1}$ et al.

\section{A SIMPLE NONLINEAR MODEL}

The model of Fig. 1 is adequate for only the crudest estimates at small displacements and is meaningless for $x>r_{0}$.

Figure 2 shows a slightly more complicated model. The electric field distorts the initially spherical atom into an ellipsoid with the fixed nucleus at its focus. The ellipsoid has constant volume, and the fastest moving point will be taken to move with the quiver velocity [i.e., its displacement is given by Eq. (2)]. The electric field from a ring of charge as shown in Fig. 2 is

$$
d^{2} E=-2 \pi r \sin \theta d r r d \theta f \frac{\cos \theta}{r^{2}} .
$$

The equation for an ellipse is

$$
\frac{1}{R}=B+A \cos \theta
$$




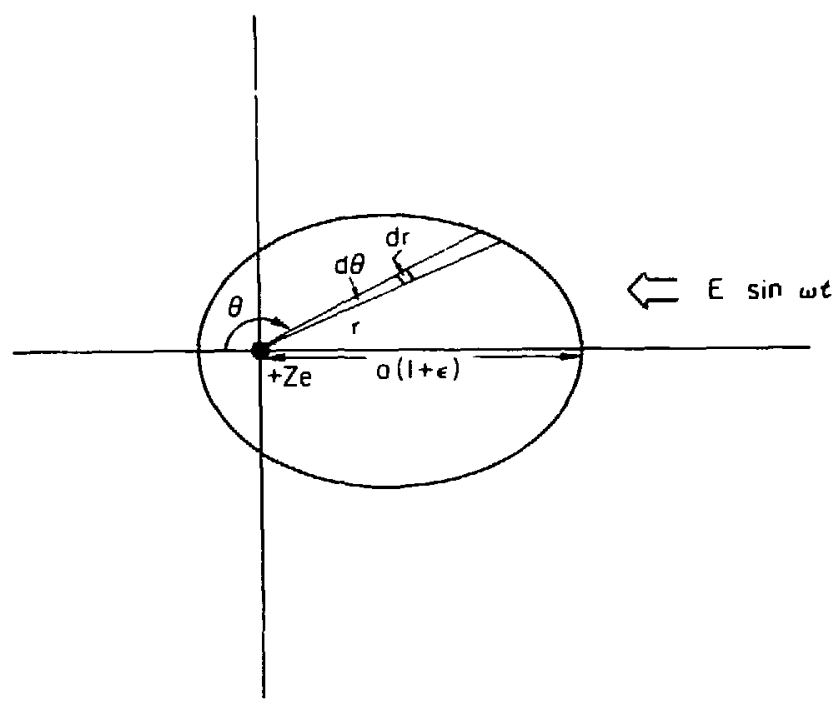

Fig. 2. Atomic electrons represented by a uniformly charged ellipsoid of constant volume. The electric field alters eccentricity.

therefore, the electric field at the nucleus is

$$
\begin{aligned}
E_{e d} & =-\int_{0}^{\pi} \int_{0}^{R} 2 \pi \rho \cos \theta \sin \theta d r d \theta \\
& =-2 \pi \rho \int_{0}^{\pi} \frac{\cos \theta \sin \theta}{B+A \cos \theta} d \theta \\
& =\left[2 \pi \rho \frac{B}{A^{2}} \ln \left(\frac{B+A}{B-A}\right)-\frac{2}{A}\right] .
\end{aligned}
$$

Recalling that the volume of an ellipsoid is $\frac{4}{3} \pi a b^{2}$, where a (along the axis of symmetry) and $b$ are the major and minor semiaxes and $b=a \sqrt{1-\epsilon^{2}}$, with $\epsilon$ the eccentricity, we find

$$
E_{e d}=\frac{3 Z e\left(1-\epsilon^{2}\right)^{2 / 3}}{2 \epsilon^{2} r_{0}^{2}}\left[\ln \left(\frac{1+\epsilon}{1-\epsilon}\right)-2 \epsilon\right]
$$

Now the fastest moving point on the ellipsoidal surface is on the axis of symmetry opposite the nucleus. Its displacement from the original sphere is

$$
x=(1+\epsilon) a-r_{0}=\left(\frac{1+\epsilon}{\left(1-\epsilon^{2}\right)^{1 / 3}}-1\right) r_{0} .
$$

Equation (12) can be inverted to give $\epsilon$ as a function of $x \geq 0$, that is,

$$
\begin{aligned}
\epsilon & =\left[2\left(1+\frac{x}{r_{0}}\right)^{3}+\frac{1}{4}\left(1+\frac{x}{r_{0}}\right)^{6}\right]^{1 / 2}-1-\frac{1}{2}\left(1+\frac{x}{r_{0}}\right)^{3} \\
& =\left\{\begin{array}{l}
\frac{x}{r_{0}}-\frac{1}{3}\left(\frac{x}{r_{0}}\right)^{2}-\frac{1}{9}\left(\frac{x}{r_{0}}\right)^{3}+\ldots, 0 \leq x \ll r_{0}, \\
1-4\left(\frac{r_{0}}{x}\right)^{3}+\ldots, x \gg r_{0}>0 .
\end{array}\right.
\end{aligned}
$$

Now, $E_{e d}(x)$ must be odd [i.e., $E_{e d}(x)=-E_{e d}(-x)$ ]. The electric field, $E_{e d}(\epsilon)$, given by Eq. (11) is odd, but $\epsilon(x)$ given by Eq. (13) is meaningless for $x<0$. Because Eq. (11) is odd, we may use $\epsilon(-x)=-\epsilon(x)$ in Eq. (11), where $x>0$. 


$$
s=a r_{0} \sin \text { it. }
$$

where $\alpha=-\frac{e E_{0}}{r_{0} m \omega^{2}}$, a dimensionless parameter. Therefore, $E_{e d}(t)$ is odd, and its Fourier series has only sine terms:

$$
b_{m}=\frac{1}{\pi} \int_{-\pi}^{\pi} E_{e d}(t) \cos m t d t=0 .
$$

Furthermore, the only time dependence of $E_{e d}$ is as a function of $\sin \omega t$; therefore, $E_{e d}(t)=-E_{e d}\left(t+\frac{\pi}{\omega}\right)$, and its Fourier series will have only odd harmonics:

$$
a_{m}=\frac{1}{\rho} \int_{-\pi}^{\pi} E_{e d}(t) \sin m t d t=\left\{\begin{array}{l}
o, \text { modd } \\
?, \text { m even }
\end{array} .\right.
$$

To evaluate this Fourier series for small values of $\alpha$, we need not undertake the tedious integrations of Eq. (17). We can approximate $\epsilon$ by the first term of Eq. (14a), which is intrinsically odd. Substituting the first term in Eq. (11) and taking a Taylor series around $x=0$, we obtain

$$
E_{c d}=\frac{Z e}{r_{0}^{2}}\left(\frac{x}{r_{0}}-\frac{x^{3}}{15 r_{0}^{3}}-\frac{26 x^{5}}{315 r_{0}^{5}}-\frac{194 x^{7}}{2835 r_{0}^{7}}-\ldots\right) .
$$

Subsituting Eq. (15) and using the multiple-angle formulas

$$
\begin{aligned}
& \sin ^{2} \theta=\frac{1}{2}(1-\cos 2 \theta), \\
& \sin ^{3} \theta=\frac{1}{4}(3 \sin \theta-\sin 3 \theta), \\
& \sin ^{4} \theta=\frac{1}{8}(3-4 \cos 2 \theta+\cos 4 \theta),
\end{aligned}
$$

we obtain

$$
\begin{aligned}
E_{e d} \cong & \frac{Z e}{90720 r_{0}^{2}}\left[97 \alpha^{7} \sin (7 \omega t)\right. \\
& +\left(-679 \alpha^{7}-468 \alpha^{5}\right) \sin (5 \omega t) \\
& +\left(2079 \alpha^{7}+2340 \alpha^{5}+1512 \alpha^{3}\right) \sin (3 \omega t) \\
& \left.+\left(-3395 \alpha^{7}-4680 \alpha^{5}-4536 \alpha^{3}+90720 \alpha\right) \sin (\omega t)\right],
\end{aligned}
$$

which verifies the second article of faith.

The electric field from electron displacement as given in Eq. (11) has a maximum value at $\epsilon \cong 0.848$ or $x \cong 1.82 r_{0}$. This means that if we are trying to drive a dipole transition, we cannot realize further gain beyond an optimum intensity. Table I gives the field strength of the first seven harmonics as a function of $\alpha$. We see that the first harmonic reaches a maximum around $\alpha=2$, which corresponds to an intensity of

$$
I \cong 3.5 \times 10^{21}\left(r_{0} / \lambda\right)^{2} W \cdot \mathrm{cm}^{-2} .
$$

'or $\lambda=193 \mathrm{~nm}$ and $r_{0}=1 \AA$, this is $I \cong 10^{15} \mathrm{~W} \cdot \mathrm{cm}^{-2}$. The higher harmonics reach maxima at progressively higher values of $\alpha$, showing that there is an optimum intensity for each.

If we take $\alpha=2$, we find from Table I that the amplitude of the first harmonic is 0.861 . Therefore, the amplification of the electric field from the laser is

$$
A=\frac{0.861}{2} \frac{Z e^{2}}{m \omega^{2} r_{0}^{3}}-1 .
$$


TABLE I. Fourier Coefficients $a_{m}$ for Electrical Field ${ }^{a}$

\begin{tabular}{cccccccc}
\hline $\mathrm{m} \alpha$ & 1 & 2 & 4 & 8 & 16 & 32 & 64 \\
\hline 1 & .6774 & {$[.8610]^{b}$} & .7743 & .4968 & .2482 & .1104 & .0430 \\
3 & .0635 & .1823 & .3201 & {$[.3343]$} & .2345 & .1271 & .0585 \\
5 & .0107 & .0473 & .1361 & {$[.2094]$} & .1907 & .1181 & .0620 \\
7 & .0032 & .0157 & .0623 & .1321 & {$[.1521]$} & .1125 & .0642
\end{tabular}

${ }^{a}$ Cofficients are for various values of $\alpha=-\frac{e E_{0}}{r_{0}} m \omega^{2}$ obtained by numerical integration.

$$
E_{e d}=\frac{Z e}{r_{0}^{2}} \sum_{\text {odd } m} a_{m} \sin (m \omega t)
$$

${ }^{b}$ Values in brackets are near the maximum, showing that there is an optimum intensity for driving each harmonic.

Again using $\lambda=193 \mathrm{~nm}$ and $r_{0}=1 \AA$, we obtain

$$
A \cong 1.2 Z-1 \text {, }
$$

which can be compared with the result from the linear model given in Eq. (6).

We now turn to the question of driving higher multipolarities in the field at the nucleus. Specifically, "ve are looking for the derivatives of the E-field along the axis of symmetry and at the focus. These are giver. by

$$
\frac{\partial^{\ell}}{\partial x^{\ell}} E_{e d}=-2 \pi \rho \int_{(B+A)^{-1}}^{(B-A)^{-1}} \int_{\cos ^{-1}\left(\frac{1}{A R}-\frac{B}{A}\right)} P_{l+1}(\cos \theta) r^{-\ell} \sin \theta d \theta d r .
$$

where $A$ and $B$ are given by Eq. (19). For $\ell=0$, we find

$$
E_{e d}=-\frac{2 \pi \rho}{A^{2}}\left[B \ln \left(\frac{B+A}{B-A}\right)-2 A\right],
$$

which is the same as Eq. (10). For $\ell=1$ we find

$$
\frac{\partial}{\partial x} E_{e d}=-\pi \rho\left[\left(\frac{B}{A}-\frac{B^{3}}{A^{3}}\right) \ln \left(\frac{B+A}{B-A}\right)+2 \frac{B^{2}}{A^{2}}-\frac{4}{3}\right],
$$

which couples to nuclear quadrupole moments and verifies the third article of faith! However,

$$
\frac{\partial^{\ell}}{\partial x^{\ell} E_{e d}}=\mathbf{0}
$$

for $\ell>1$. There is no coupling to the nucleus for octupole or higher moments. This is obvious from the symmetry of the ellipsoid: to have an octupole coupling, it would have to assume a pear shape at some place in its cycle.

Now $\frac{\partial}{\partial x} E_{e d}(t)=\frac{\partial}{\partial x} E_{e d}\left(t+\frac{\pi}{\omega}\right)$; therefore, the quadrupole will couple only to even harmonics. In terms of eccentricity, Eq. (26) can be written

$$
\frac{\partial}{\partial x} E_{e d}=\frac{Z e}{r_{0}^{3}}\left[\frac{3\left(1-\epsilon^{2}\right) \ln \left(\frac{1+\epsilon}{i-\epsilon}\right)+4 \epsilon^{3}-6 \epsilon}{4 \epsilon^{3}}\right]
$$


which is an even function of $\epsilon$.

Taylor expanding Eq. (28) around $\epsilon=0$ and substituting the first term of Eq. (14a) for $\epsilon$, we find

$$
\frac{\partial}{\partial x} E_{e d} \cong-\frac{\Sigma e}{r_{0}^{3}}\left(\frac{x^{2}}{5 r_{0}^{2}}+\frac{3 x^{4}}{35 r_{0}^{4}}+\frac{x^{6}}{21 r_{0}^{6}}+\frac{x^{8}}{33 r_{0}^{8}}+\ldots\right) .
$$

By substituting Eq. (15) and using the Inultiple-angle formulas of Eq. (19), we can obtain an expression that gives the even harmonics for very small $\alpha$. Of more interest is the limit of large $\alpha$. We note that from Eq. (28),

$$
\lim _{\epsilon \rightarrow 1} \frac{\partial}{\partial x} E_{e d}=-\frac{Z e}{2 r_{0}^{3}}
$$

Re-expressing Eq. (28) using Eq`. (14), (15), and (19), we have

$$
\lim _{\alpha \rightarrow \infty} \frac{\partial}{\partial x} E_{e d}=-\frac{Z e}{2 r_{0}^{3}}\left\{\begin{array}{l}
0: t=\frac{n \pi}{\omega} \\
1: \text { otherwise }
\end{array} .\right.
$$

Because Eq. (28) is even,

$$
a_{n}=\mathbf{0}
$$

(i.e., all sine terms of its Fourier series vanish, and all harmonics must be in phase or $180^{\circ}$ out of phase with the driving radiation). Furthermore,

$$
\lim _{\alpha \rightarrow \infty} b_{m}=\left\{\begin{array}{l}
0 ; m \neq 0 \\
2 ; m=0
\end{array}\right.
$$

all oscillatory components of the quadrupole coupling vanish at high intensity.

Because the oscillatory components vanish both at $\alpha=0$ and as $\alpha$ approaches infinity, they must have a maximum value in between. Thus, there is an optimum, intensity for driving eack harmonic, as illustrated in Table II.

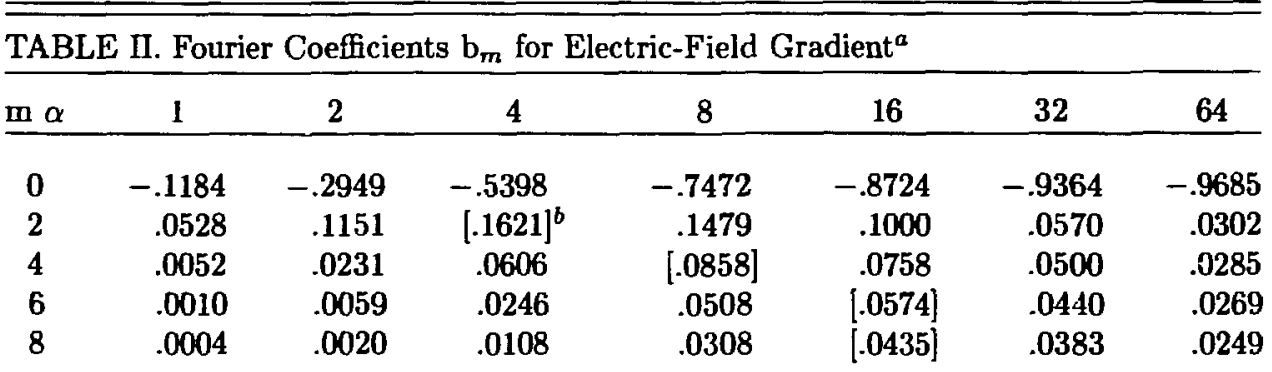

${ }^{a}$ Cofficients are for various values of $\alpha=-\frac{e E_{0}}{1_{0}} m \omega^{2}$ obtained by numerical integration.

$$
\frac{d}{d x} E_{e d}=\frac{Z e}{r_{0}^{3}}\left[\frac{1}{2} b_{0}+\sum_{e v e n m} b_{m} \cos (m \omega t)\right]
$$

${ }^{b}$ Values in brackets are near the maximum, showing that there is an optimum intensity for driving each harmonic. 


\section{COMMENTS ON THE U ${ }^{235}$ EXPERIMENT}

This experiment intends to couple the 15th harmonic of $\frac{\partial^{3}}{\partial x^{2}} E_{e d}$ to the nuclear electric octupole. As shown in Tables I and II, higher harmonics generally drop off rapidly. Furthermore, in the simple nonlinear model the coupling of $\frac{\partial}{\partial x}=E_{e d}$ to typical nuclear quadrupoles is greatly reduced from the coupling of $E_{e d}$ to typical nuclear dipoles, and the coupling to octupoles, as well as coupling to all higher multipoles, disappears entirely. For a gamma-ray laser, we would like to transfer from the storage state by a dipole transition and hopefully at the driving frequency, not at a harmonic. This is much easier.

Therefore, success of the $U^{235}$ experiment should be interpreted as exceedingly encouraging for development of a gamma-ray laser, and inability to detect any excitation should not be discouraging.

\section{REFERENCE}

1. Charles K. Rhodes, Science 229 (4720), 1345 (1985); K. Boyer and C. K. Rhodes, Phys. Rev. Lett. 54 (14), 1490-1493 (1985). 